\title{
Button Sequestrum Sign Due to Osteomyelitis of the Mandible Secondary to Malignant Infantile Osteopetrosis
}

\author{
Evangelia F. CHRISTODOULOU, Georgios NISTIKOULIS, Savas P. DEFTEREOS
}

Department of Radiology, Democritus University of Thrace -

Medical School/University General Hospital of Alexandroupolis, University Campus Dragana, 68100, Alexandroupolis, Greece

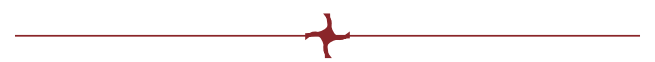

\begin{abstract}
CABSTRACT
The button sequestrum sign is demonstrated in a number of medical conditions and refers to a lesion of devascularised bone which is surrounded by lucency. Although it may be difficult to arrive at a single diagnosis based on this sign, the combination of clinical and paraclinical findings, patient's medical history and imagistic presentation of this sign can lead to a great specificity in chronic osteomyelitis, even if osteomyelitis is accompanied by osteopetrosis, as in the present case.
\end{abstract}

Keywords: button sequestrum, osteomyelitis, osteopetrosis, computed tomography, paediatric.

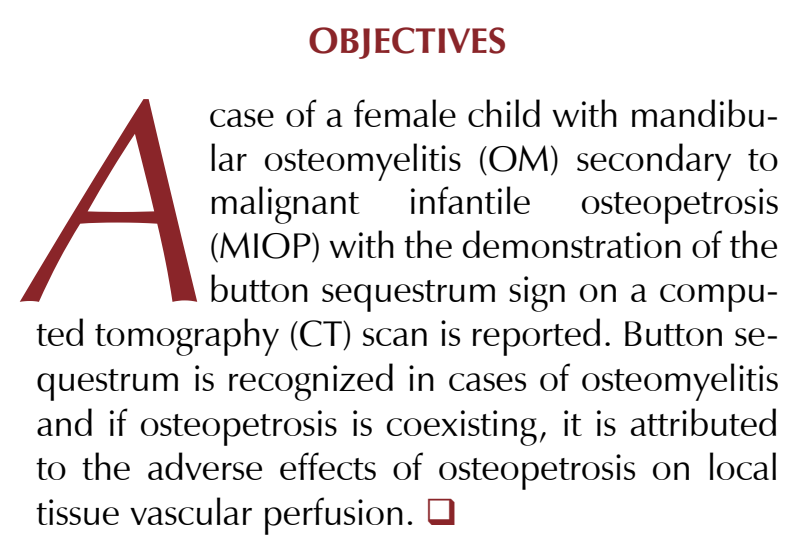

\section{MATERIALS AND METHODS}

n 11-year-old female patient was referred to the Radiology Department with past history of mandible OM for follow-up evaluation with a CT scan of the head and face. Past medical history revealed the diagnosis of MIOP, which is a rare recessively inherited autosomal osteosclerosing type of skeletal dysplasia. The clinical suspicion of MIOP was made after the physical examination by a paediatric consultant. Growth retardation and dysmorphic facial features (prominent frontal bossing, depressed nasal bridge and wide opened anterior fontanelle) were revealed. No clinical symptoms were referred by then. The patient underwent bone marrow biopsy and specimens revealed the presence of all bone marrow cell lines, megakaryocytes and a great number of hematogones. Also, a number of surgical approaches for tooth extrac-

\footnotetext{
Address for correspondence:

Evangelia F. Christodoulou, MD, cMSc, Radiology Resident

Department of Radiology, Democritus University of Thrace - Medical School/University General Hospital of Alexandroupolis, University Campus Dragana, 68100, Alexandroupolis, Greece

Tel: +306980383822, Email: evelyn.christod@gmail.com
}

Article received on the the $23^{\text {rd }}$ of July 2021 and accepted for publication on the $29^{\text {th }}$ of September 2021

Maedica $\mid$ A Journal of Clinical Medicine, Volume 16, No. 3, 2021531 


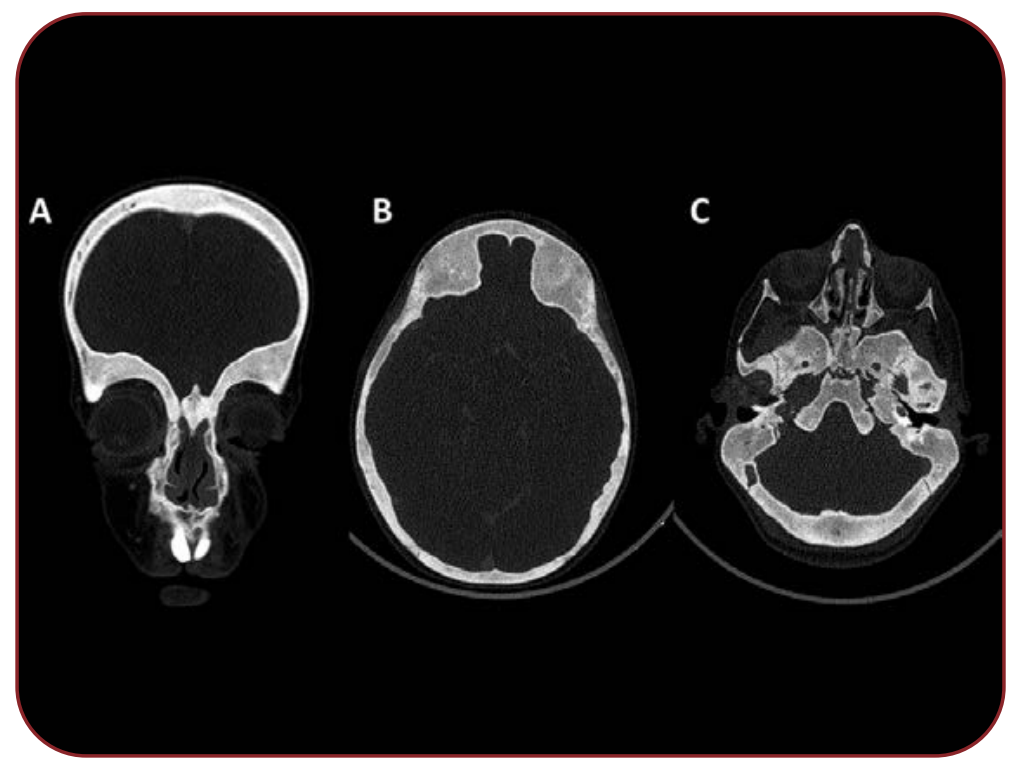

FIGURE 1. Coronal (A) and axial (B \& C) CT-images in bone window setting of an 11-year-old female patient. Facial-CT revealed frontal and ethmoid sinuses aplasia/hypoplasia, hypertelorism and skull-bones thickening with high-attenuation inner table, broad, low-attenuation diploic space, less high-attenuation outer table. Also, no mastoid air cells are depicted (area of petrous bone bilateral). The findings are compatible with osteopetrosis.

tions were performed. After these surgical approaches histopathologically checked specimens from the alveolar process of the mandible, at the 46-tooth level revealed oral mucosal ulceration, inflammatory papillary hyperplasia, inflammatory fibrous hyperplasia and other reactive lesions. Histologic examination of the removed teeth was delayed due to desalination processes.

FIGURE 2. Facial CT scan of the same patient, axial image. A destructive lucent lesion involving right mandibular body at the inner site is depicted. Centrally to this lesion, a sclerotic small bone tissue surrounded by lucency is revealed. The findings are compatible with "button sequestrum" of the mandibular bone.

\section{RESULTS}

—acial CT scan revealed findings which were compatible with osteopetrosis, including delayed teeth eruption, frontal and ethmoid sinuses aplasia/hypoplasia, hypertelorism and skull bone thickening with high attenuation appearance of the inner table, broad and low attenuated diploic space, less high attenuated outer table (Figure 1). Furthermore, a destructive lucent lesion involving the right mandibular body at the inner site of 45 and/or 46-teeth was depicted. Centrally to this lesion, there was a sclerotic, small bone tissue which was surrounded by lucency and which was compatible with button sequestrum (Figure 2).

\section{DISCUSSION}

B utton Sequestrum sign is not a pathognomonic radiographic feature as it is presented in many diseases. It is classically described in osteomyelitis but also in eosinophilic granuloma and occasionally in fibrosarcoma and lymphoma $(1,2)$. Other entities with similar radiological appearance include the calcified intraosseous lipoma, tuberculous osteitis/osteomyelitis, radiation necrosis, metastatic carcinoma, fibrous dysplasia, epidermoid and dermoid cyst, osteoid osteoma, haemangioma, meningioma, etc $(1,2)$.

Haematopoietic stem cell transplantation (HSCT) tends to be the only treatment with significant clinical results (amelioration of radiographic bone lesions is an important indicator of a successful HSCT therapy), while gene-based therapies are considered to be promising. Treatment with other therapeutic alternatives (corticosteroids, colony-stimulating factor 1 , vitamin $\mathrm{D}$ and interferon- $\gamma$ ) has been proved inefficient as resistance occurs (3). If this medical condition be untreated, it may result in death. In our case, the patient was being treated in another medical centre, so detailed information about the treatment protocol was not being obtained.

In osteomyelitis cases, the lucent area is caused by infectious organisms, which destroy the bone tissue (in eosinophilic granuloma, it is caused by erosive accumulations of histiocytes) (1). The destroyed area is replaced by purulent and granulation tissue. The central opacity represents the residual bone tissue after avascular necrosis (1). Radiographs can also demonstrate this sign. MIOP 
is an extremely rare disorder that has characteristic findings such as the generalized sclerosis of the bone skeleton as a result of the reduced activity of the osteoclasts ("bone in bone" appearance), macrocephaly with frontal bossing and hematologic abnormalities (bone marrow failure and extramedullary hematopoiesis) (3). Osteomyelitis is a well-documented complication of osteopetrosis. order to shrink the differential diagnosis, physicians have to consider any demographic data, the medical history, physical examination, laboratory findings and the imaging results obtained through radiology procedures of their patients. Different specialties should be involved in such a case for a more favourable outcome.

\section{CONCLUSION}

The button sequestrum is an uncommon ma-

Conflicts of interest: none declared.

nifestation of multiple clinical disorders. In Financial support: none declared.

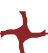

\section{R}

1. Krasnokutsky MV. The button sequestrum sign. Radiology 2005;236:1026-1027. doi:10.1148/radiol.2363031294.

2. Helms CA. Computed tomography and plain film appearance of a bony sequestrum: significance and differential diagnosis.

Skeletal Radiol 1987;16:117-120.

doi:10.1007/bf00367758
3. Machado CDV, Siquara da Rocha MCB, Telles PDS. Infantile osteopetrosis associated with osteomyelitis. BMJ Case Rep 2015;2015:bcr2014208085. doi:10.1136/bcr-2014-208085. 